\title{
Laparoscopic management of ectopic pregnancies at unusual sites
}

\author{
Sunita Kumar* \\ Department of Obstetrics and Gynecology, Sir Ganga Ram City Hospital, New Delhi, India
}

Received: 01 July 2015

Revised: 17 August 2015

Accepted: 19 August 2015

\author{
*Correspondence: \\ Dr. Sunita Kumar, \\ E-mail: drsunitakumar@hotmail.com
}

Copyright: () the author(s), publisher and licensee Medip Academy. This is an open-access article distributed under the terms of the Creative Commons Attribution Non-Commercial License, which permits unrestricted non-commercial use, distribution, and reproduction in any medium, provided the original work is properly cited.

\begin{abstract}
Ruptured ectopic pregnancies are the most common life threatening emergencies in the obstetrical practice. The tubal ectopics are the most common, but increasing infertility treatments and ART procedures may lead to a rise in the other sites of ectopic also. In the first case of ectopic pregnancy in the ampullary region, the tube was forming a TO Mass and salpingectomy of this damaged tube was not possible because of the adhesions all around and hence ectopic was removed after salpingostomy. The second case with ectopic in the isthmic region with impending rupture was dealt with salpingectomy. The third a cornual pregnancy presented with severe anemia and peripheral vascular collapse, and the ectopic was removed after cornustomy. The fourth case of an ovarian pregnancy again presented with pain abdomen and hemodynamic instability and ovariectomy with removal of the ectopic and some ovarian tissue was done. Ruptured ectopics with their varied and unexpected presentation are great challenges for the minimal invasive procedures in a hemodynamicaly unstable patient. Advancement in the diagnostic and minimal invasive techniques has led to a rapid decline in the mortality and morbidity in such cases. The purpose of reporting these cases are to show the varied and life threatening presentations of these ectopics in unsuspected patients.
\end{abstract}

Keywords: Unusual sites of ectopic pregnancies, Laparoscopic management

\section{INTRODUCTION}

Ectopic pregnancies occur when the gestational sac is not implanted at the normal site in the uterine cavity but at any other place, which could be the tube, ovary, cervix, uterine cornu, or scar of the previous section. The reason for ectopic pregnancies are varied, it could be PID, tubal damage, intra uterine devices or ART.

Tubal ectopics are the most common with an incidence of $95-96 \%$. The fallopian tube is about $10 \mathrm{~cm}$ long with a variation of 7-14 cm and is structurally and functionally divided into different portions. The difference in the structure of the different portions of the tube is what dictates the type of surgical management. Most of the pregnancy in the fimbrial and infundibulum region of the tube may end in tubal abortion. The ectopic in the ampulla can grow to a considerable size without tubal rupture and hemoperitoneum, but ectopic in the isthmic and cornual region can lead to disastrous effects.

Ovarian ectopic is quite uncommon with an incidence of $1-3 \%$, and there is a tendency towards an increase in the incidence due to the increasing number of infertility treatment and ART procedures.

Interesting cases of ectopic pregnancies at unusual sites, in the tubal ampullary region of a tube forming a TO mass, tubal isthmic region, the cornual pregnancy, and the ovarian ectopic is described with their presentation and management along with the review of literature. 


\section{CASE REPORT}

\section{Pregnancy in the ampulla (in a TO Mass)}

A young primigravida presented with almost 8 weeks amenorrhea, pain abdomen and a positive pregnancy test. She had a history of being treated for tuberculosis in the childhood. On examination the uterus was soft and bulky with mass right adenexa. Patient was sent for an ultrasound which revealed an unruptured right ectopic pregnancy. She was taken up for laparoscopic management.

On laparoscopy there seemed to be no blood in the abdominal cavity but the tube along with the ectopic and the ovary was forming a TO mass which was adherent to the gut and omentum. After adhesiolysis, incision was given over the ampullary region and the ectopic gestation separated from the surrounding tubal wall by the suction canula and removed in total. After separating the tube it was seen that the fimbrial end adjacent to the uterine wall had been bleeding into the uterus with clots destroying the uterine wall from outside. The abdomen was cleared of all the clots and adhesions as far as possible and a careful D \& C done so as to avoid perforation through the damaged area. Patient had a normal recovery after the surgery (Figure 1a, 1b \& 1c).

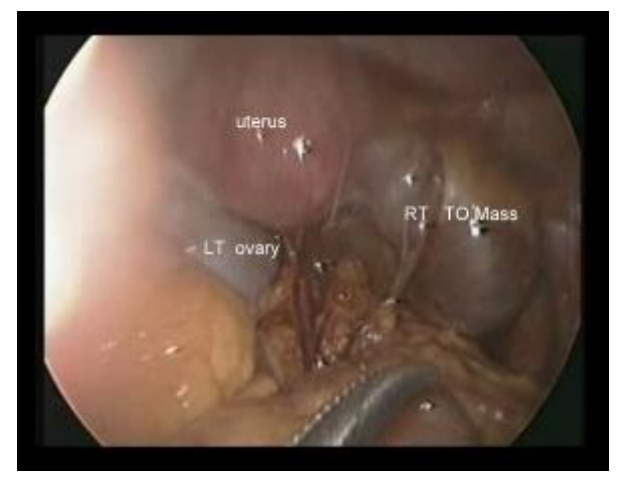

Figure 1a: Laparoscopic view of ectopic pregnancy in the right ampullary region in a TO mass with the gut and omentum adherent to it.

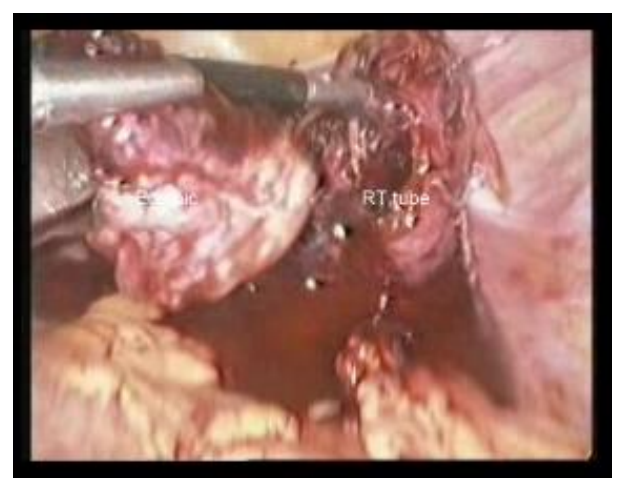

Figure 1b: Laparoscopic removal of the ectopic after salpingostomy in the right ampullary portion of the TO mass.

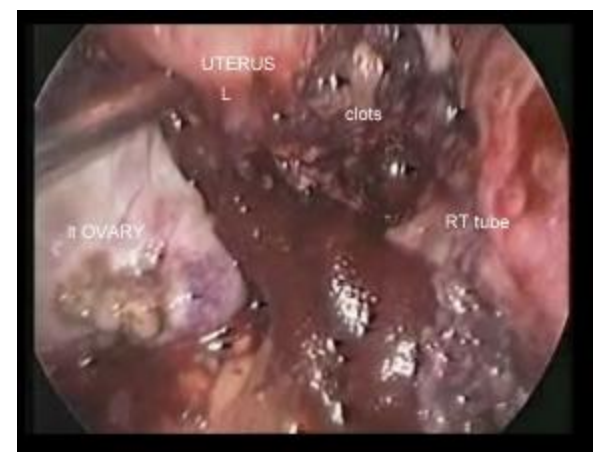

Figure 1c: Right posterolateral surface of the uterus eroded and damaged by the bleeding right tube and clots, post-surgery picture showing spongistron and drain left in situ.

The incidence of ectopic in the ampulla is $70 \%$.This portion of the tube has a thick muscularis between the tubal serosa and inner lumen. Ectopic in this region grows outside the lumen into this thick muscular area and the pregnancy can attain a considerable size without rupturing.

A TO mass is generally formed due to severe pelvic infections of which tubercular is the most common. Tubercular infections generally damage the tube and fimbria and in chronic cases lead to formation of TO masses. They are usually known to cause infertility due to tubal blockage, but this is an interesting case of ectopic pregnancy in the TO mass which was probably due to patent but damaged tube where the ectopic got implanted in the ampulla and kept on bleeding through the fimbrial end, there was no tubal rupture in the ampullary region and the bleeding was concealed between the uterine wall and the mass destroying the uterine wall.

\section{Ectopic pregnancy in the isthmic portion of the tube}

This patient was Para 1 with a previous normal delivery who now presented with amenorrhea of 6 weeks and pain abdomen. Her pelvic examination showed a mass right adenexa and ultrasound revealed an ectopic gestation with a dilated tube. On laparoscopy there was ectopic in the isthmic portion of the tube with signs of impending rupture. Right Salpingectomy was done and the tube along with the ectopic retrieved through the $10 \mathrm{~mm}$ port (Figure 2a \& 2b).

The isthmic portion of the tube has a thin muscularis layer and is not only shorter in length, but due to the proximity to the uterus is very vascular. The incidence of ectopic in this region is around $12-15 \%$. Pregnancy in this region continues to grow in the lumen of the tube and hence destroys the lumen as the pregnancy grows in size. With a rupture of such an ectopic one should expect massive and life threatening hemoperitoneum. The surgery of such an ectopic is either complete or segmental salpingectomy, taking care of the vessels by bipolar cautery. If removal of the ectopic is done by 
linear salpingostomy in an isthmic pregnancy, there is an increased risk of tubo-peritoneal fistula formation, as well as bleeding during surgery due to the increased vascularity.



Figure 2a: Ectopic pregnancy in the right isthmic region of the tube.



Figure 2b: Showing the extension of the ectopic gestation in the right isthmic region with impending rupture and increased chances of tuboperitoneal

fistula if salpingostomy is attempted.

\section{Ectopic pregnancy in the cornual portion of the tube}

A 29 year old lady was admitted in the emergency with pain abdomen, loose motions and vomiting. She was treated with antibiotics, I/V fluids and other supportive medicines. Her pain persisted and so she was advised for an ultrasound, to every one's surprise the ultrasonologist raised an alarm as the abdomen had massive hemoperitoneum and she was referred to the gynecologist and general surgeon .On detailed history she revealed she had taken abortifacient drugs for termination of early pregnancy, her hemoglobin had dropped to $4 \mathrm{gm} \%$, and she was going into peripheral vascular collapse, so she was taken for laparoscopy with a provisional diagnosis of ruptured ectopic. On laparoscopy after identifying the bleeding left cornual pregnancy the bleeding was stopped from the medial and the lateral feeding vessels of the tube by bipolar cautery before attempting to remove the ectopic .The tube was opened through the bulging site of the ectopic and products of gestation removed, abdomen cleared and the tube left in situ as any chance of further hemorrhage had to be avoided. She was given blood and blood products during surgery. Post operatively her BHCG was checked for any remnant trophoblastic tissue but it was normal. Patient had a speedy recovery without any postoperative complications (Figure 3a, 3b \& 3c).

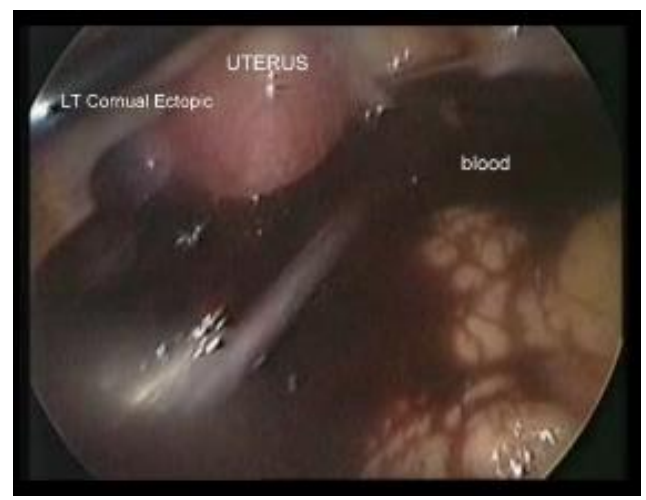

Figure 3a: Laparoscopic view of left cornual pregnancy with massive hemorrhage.

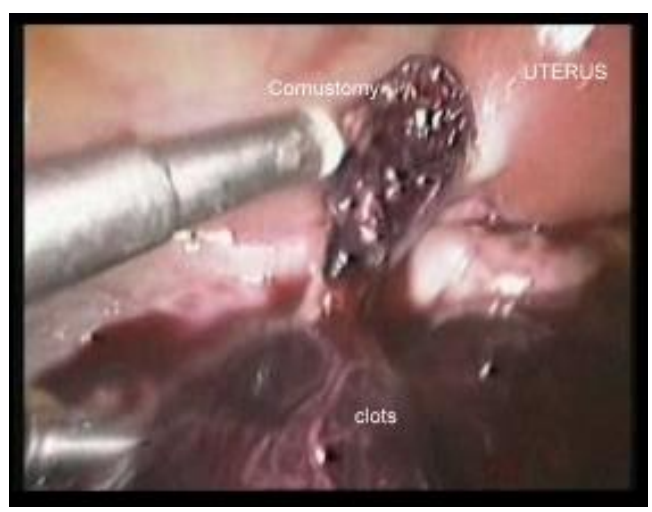

Figure 3b: Left cornustomy to remove the products of gestation.

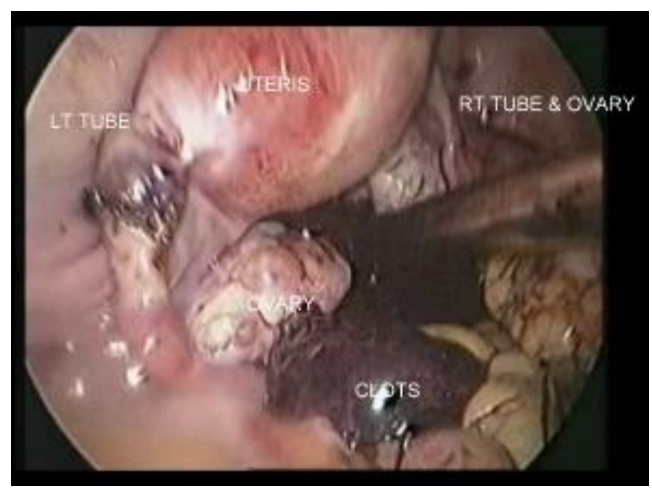

Figure 3c: Laparoscopic view post-surgery to show complete hemostasis of the cornual region without suturing.

Cornual or interstitial pregnancy with the pregnancy implanted into the portion of the tube adjacent to the uterus and entering the uterine cornu can grow to a considerable size in the muscular wall of the uterus. 
Removal of pregnancy in this area may require an incision over the uterine cornu with suturing after removal of the products of gestation. In this case because the ectopic was not growing into the uterine musculature, it could be evacuated thru the lumen. The incidence of ectopic in the cornual/interstitial region is $2-3 \%$.

\section{Ectopic pregnancy in the ovary}

A nulliparous 26 year old lady came to the casualty with pain abdomen, there was no history of amenorrhea but she had an ultrasound report done the same morning for pain abdomen which showed right ovarian cysts and left ovarian mass. Patient complained of dizziness and was hemodynamically unstable. Keeping ectopic pregnancy in mind her pregnancy test was done which was strongly positive. She was taken for laparoscopy in view of her clinical condition, ultrasound and pregnancy test. On laparoscopy the abdomen showed plenty of blood and blood clots with both the tubes normal, and a ruptured left ovarian pregnancy. The right ovary was enlarged with a corpus luteal cyst. The products of gestation along with some ovarian tissue was removed from the left ovary and hemostasis achieved, corpus luteal cystectomy was done on the right side. The histopathology report confirmed ovarian pregnancy of the left side and corpus luteal cyst of the right side (Figure 4a, 4b \& 4c).

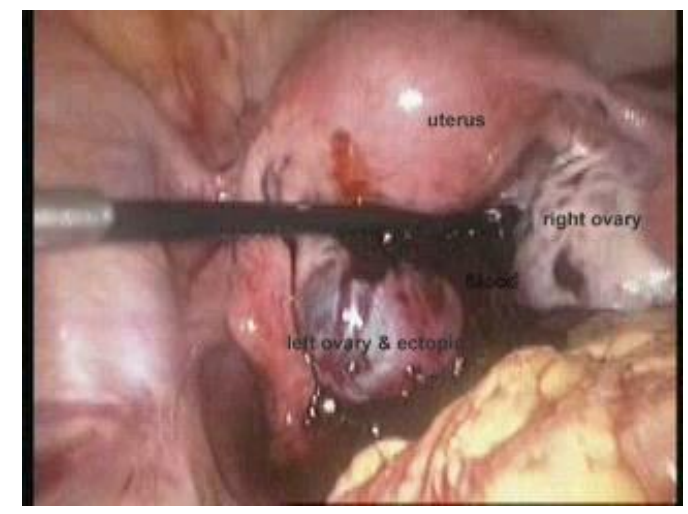

Figure 4a: Laparoscopic view of bleeding left ovarian ectopic with right corpus luteal cyst.

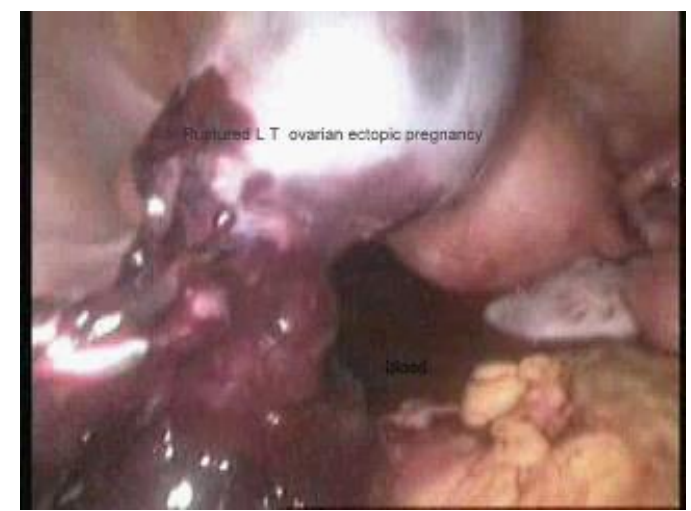

Figure 4b: Laparoscopic removal of clots and product of gestation from the left ovary.

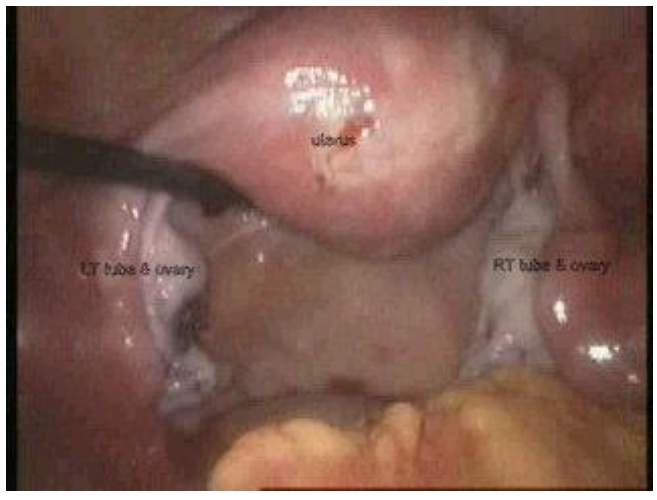

Figure 4c: Laparoscopic view post-surgery of both the tubes and the ovaries.

Ovarian pregnancies are rather rare with an incidence of $1-2 \%$. The ovary being such a vascular structure tends to bleed more. Ovarian ectopic can only be suspected clinically, but the diagnosis is usually always during surgery which has to be confirmed by histopathology. Ovarian pregnancy needs the Spiegelbergs criteria to be diagnosed which states that a) the gestational sac should be located in the region of the ovary, b) the ectopic should be attached to the uterus by the ovarian ligament, c) ovarian tissue in the wall of the gestational sac should be confirmed by histopathology, d) tube on the involved side should be intact.

With the advancement in the diagnostic facilities one can diagnose an ovarian pregnancy by a wide echogenic ring on the ovary, frequently with yolk sac and fetal parts on 2D \& 3D ultrasound pre operatively also, although the color Doppler may not be very useful due to the increased vascularity.

\section{DISCUSSION}

Advancement and development acquiring newer techniques and equipments for better diagnosis and treatment is the goal of every individual as well as institution. Modernization in the field of medicine as a whole has led to evolvement in the treatment of practically all the diseases and ailments.

Treatment of ectopic pregnancies has also evolved and changed. Not all the ectopic pregnancies need surgery but a judicious and timely management can save the patient from future morbidity and fertility problems where surgery is the only option. The first ectopic surgery was done by Dr. Robert C Trait in 1883 which was not successful, (ectopic pregnancy foundation registered charity No. 1122286) but since then the surgical approach has only improved and it would not be incorrect to say that any woman who manages to reach the hospital should not face mortality or severe morbidity due to an ectopic pregnancy.

Rare case like a pregnancy in a TO mass deserves mentioning, as pregnancy with such damaged tube is not 
expected initially and the bleeding into the uterine wall which was not picked up on ultrasound would have led to a disastrous uterine perforation and bleed if evacuation or a D \& C was tried without an ultrasound by a general practitioner. The review of literature has shown ectopic pregnancy growing to considerable size in the ampullary region followed by a Successful management ${ }^{1,2}$ but no case report of ectopic in a TO mass with erosion of the uterine wall could be picked up.

Pregnancy in the isthmic portion of the tube has to be dealt with segmental resection or salpingostomy depending on the case and the patient's desire for future fertility. Tubal anastomosis after segmental salpingectomy is a better option than salpingostomy in such cases, but an attempt to do it in the same sitting may add on to the chances of infection due to bleeding and edema. $^{3-5}$

Cornual pregnancies are usually dealt with cornutomy, cornual resection or incision and suturing of the uterine musculature after removal of the products of gestation or simple salpingectomy with removal of part of the uterine cornu and all the products of gestation depending on the case and the feasibility. Salpingectomy with removal of the ectopic from the cornu could cause more than usual bleeding and many surgeons have used vasopressin to decrease the bleeding during such surgeries. ${ }^{6,7}$

Treatment for ovarian pregnancy could be ectopic pregnancy enucleation, corpus luteal cystectomy, wedge resection of ovary, ovariectomy or oopherectomy depending on the case and the patient's requirements. ${ }^{8-10}$ Ovarian pregnancies difficult to diagnose preoperatively has increased chances of hemorrhage due to the vascularity of the ovary.

A decision for Salpingectomy or salpingostomy depends on the location of the ectopic in the tube as well as parity of the patient. It has been seen that suturing of the tube after salpingostomy does not improve the rate of pregnancy, in fact it is noted that pregnancy occurs sooner when the tube heals by secondary intention. ${ }^{11}$ Various studies have shown that salpingostomy does not significantly improve the fertility rate, or the rate of the ongoing pregnancies. On the contrary the rate of repeat ectopic is higher, chances of persistence of trophoblastic tissue are higher after salpingostomy, and risk of conversion of salpingostomy to salpingectomy due to persistent bleeding of the tube is $20 \% .^{12}$ RCOG guidelines suggest salpingostomy should not be preferred if the contra lateral tube is normal and healthy and should be done only after the patient has been explained about the consequences and understands its merits and demerits. $^{13}$

\section{CONCLUSION}

The ectopic pregnancies would top the obstetrical emergency list with their varied life threatening presentations. Experts in every field are challenging the old text book teaching and taking up minimal invasive route for most of the surgeries. Ruptured ectopic is no longer a contraindication for laparoscopic approach, in fact it gives you a better view with better chances for future fertility besides an early and a rapid recovery. The purpose of reporting these cases was to show how differently and unsuspectedly these ectopic pregnancies land up in emergencies, but still most of them can be treated laparoscopically. The medical management of early pregnancies is a great step in doing away with the unwanted pregnancies without any surgical interference and cost incurred, but with the distribution of these drugs without a prior ultrasound or proper vaginal examination, could only lead to rise in the number of such emergencies.

\section{Funding: No funding sources \\ Conflict of interest: None declared \\ Ethical approval: Not required}

\section{REFERENCES}

1. Sachan R, Gupta P, Patel ML. Second trimester unruptured ampullary ectopic pregnancy with variable presentation, report of two cases. Int J Case Rep Images. 2012;3(8):1-4.

2. Khalil MM, Badran E, Ramadan MF, Elkarim SA, Shazly M, Kharing Ali M, et al. An advanced second trimester tubal pregnancy: case report. Middle East Fertil Soc J. 2012;17(2):136-8.

3. Morandans S. An unruptured isthmic ectopic pregnancy: a case report. Zahedan Jres Med Sci (ZJRMS). 2013;15(9):88-9.

4. Akihiro Takeda, Shucichi Monabe, Takshi Mitsui Hiromi Nakamura. Spontaneous ectopic pregnancy occurring in the isthmic portion of the remnant tube after ipsilateral adenexectomy: report of two cases. $\mathbf{J}$ Obstet Gynecol Res. 2006;32(2):190-4.

5. Bahreh Samiei-Sarir, Christopher Diehm. Recurrent ectopic pregnancy in the tubal remnant after salpingectomy. Case Rep Obstet Gynecol. 2013;2013:753269.

6. M. Sami Walid, Richard L. Heaton. Diagnosis and laparoscopic treatment of cornual ectopic pregnancy. Ger Med Sci. 2010;8:16.

7. Kelekei S, Desdiciogly R, Ceyhan Ozdemir A, Eris S, Cetrikaya Z, Aydogmus H. Laparoscopic management of two unusual ectopic pregnancies. OA Case Rep. 2014;3(2):17.

8. Tehrani HG, Hamoush Z, Ghasemi M, Hasheri L. Ovarian ectopic pregnancy: a rare case. Iran J Reprod Med. 2014;12(4):281-4.

9. Shrshtha A, Chawla CD, Shreshtha RM. Ruptured primary ovarian pregnancy: a rare case report. Kathmandu Univ Med J (KUMJ). 2012;10(39):76-7.

10. Tinelli A, Huelist G, Malvesi A, Tinelli R. Laparoscopic management of ovarian pregnancy. JSLS. 2008;12(2):169-72.

11. Tulandi T, Guralnick M. Treatment of tubal ectopic pregnancy by salpingostomy with or without tubal 
suturing and salpingectomy. Fertil Steril. 1991;55(1):53-5.

12. Mol F, van Mello NM, Strandell A, Strandell K, Jurkovic D, Ross J, et al. Salpingostomy versus Salpingectomy in women with tubal pregnancy (ESEP) study. An open label multicentric randomized control trial. Lancet. 2014;383(9927):1483-9.
13. RCOG guidelines. Tubal pregnancy, management (Green-top Guideline No 21), May 2004. Available at: https://www.rcog.org.uk/en/guidelines-researchservices/guidelines/gtg21\%. Accessed November 2010.

Cite this article as: Kumar S. Laparoscopic management of ectopic pregnancies at unusual sites. Int J Reprod Contracept Obstet Gynecol 2015;4:1555-60. 